\title{
Oocyte Cryopreservation in Emergency Situations: Perspectives and Reality
}

Authors:

Disclosure:

Acknowledgements:

Received:

Accepted:

Keywords:

Citation:

\section{*Taisiia Yurchuk,, Maryna Petrushko,, ${ }^{1}$ Barry Fuller, ${ }^{2}$ Anatoliy Goltsev ${ }^{1}$}

1. Institute for Problems of Cryobiology and Cryomedicine (IPC\&C) of the National Academy of Sciences of Ukraine and United Nations Educational, Scientific and Cultural Organization (UNESCO) Chair in Cryobiology, Kharkiv, Ukraine

2. University College London Division of Surgery and Interventional Science, Royal Free London National Health Service Trust, Royal Free Campus, London, UK

*Correspondence to taisiya.yur@gmail.com

The authors have declared no conflicts of interest.

This review was generated as a result of discussions between the authors, and supported by the UNESCO Chair in Cryobiology based at the IPC\&C of the National Academy of Sciences of Ukraine (NASU). Authors are grateful to Evgeniia Pushkova, head of the Information Department of the IPC\&C of the NASU, for support in the paper processing.

04.04 .20

05.06 .20

Cancer, immature oocyte, oocyte in vitro maturation, urgent fertility preservation, vitrification.

EMJ Repro Health. 2020;6[1]:54-62.

\section{Abstract}

Increased incidence of global recorded cancer, unforeseen circumstances in assisted reproductive technology, a pandemic situation, and surgical interventions which can cause impairment of the reproductive system all necessitate urgent fertility preservation. Unfortunately, the application of successfully developed methods for oocyte and embryo cryopreservation is not possible in some situations because of contraindications for inducing superovulation, inability to delay other treatments, or in the case of prepubertal patients; in these cases, cryopreservation of ovarian tissue may be an alternative method. Despite current achievements in ovarian tissue low-temperature preservation, only 130 children have been born using this method. Further development of this technique and methods for in vitro maturation of immature oocytes, following their cryopreservation and use in assisted reproductive technology, as well as a differentiated approach for the selection of mature oocytes obtained without preliminary superovulation are needed. This review outlines the modern achievements and future prospects of female fertility preservation in emergency situations by cryopreservation of oocytes with different quality and maturity states.

\section{INTRODUCTION}

The need to preserve women's fertility urgently arises when unforeseen circumstances occur in assisted reproductive technology (ART), such as when there is no possibility to fertilise aspirated

oocytes, in a pandemic situation, when there are high risks of complications during pregnancy for patients undergoing infertility treatment, following surgical interventions that can cause impairment of the reproductive system, and in cancer patients requiring immediate 
chemotherapy with gonadotoxic effects., ${ }^{1,2}$ Annually, there is a global increased incidence of cancer so development of methods for preservation of fertility in patients after successful cancer treatment is both relevant and important. ${ }^{3}$ There are currently several fertility conservation strategies. ${ }^{4}$ The first strategy is the 'gold standard' of emergency ovarian stimulation followed by embryo or oocyte cryopreservation. However, this method can be inappropriate for single women refusing sperm donation and for prepubertal girls. ${ }^{5}$ Mature oocyte retrieval is associated with hormonal stimulation of superovulation, contraindicated in hormone-dependent tumours. ${ }^{6}$ Moreover, ovarian stimulation protocol delays the initiation of cancer therapy which may increase the likelihood of relapse. ${ }^{7}$ The second strategy is ovarian tissue extraction followed by ovarian tissue freezing and autotransplantation or in vitro maturation (IVM), and is currently considered experimental. The third strategy includes ovarian protection techniques such as oophoropexy but their efficacy is debatable. Further study and development of the experimental strategies is relevant, because they are an alternative for those strategies that cannot be applied for female fertility preservation.

To date, cryopreservation of ovarian tissue is under development and a little more than 130 children have been born following its use. ${ }^{8}$ In addition, the use of autotransplantation in patients who have recovered after cancer may cause metastasis unless cases are carefully selected. Cryopreservation of individual follicles could solve this problem; however, after thawing it is necessary to carry out in vitro growth which has not yet led to the production of mature oocytes. ${ }^{9}$

The absence of preliminary ovarian stimulation means that retrieval of immature oocytes will require further IVM. There is no difference in the number of oocytes or their IVM rates relating to the phase of the menstrual cycle at which oocyte retrieval is performed, and IVM can be a promising tool for patients with breast cancer seeking urgent oocyte cryopreservation. ${ }^{10}$ But, even in cases where retrieved oocytes are mature, they may have morphological abnormalities, resulting from intrinsic or extrinsic factors, that can impact on the cryopreservation efficacy and further fertilisation outcomes." In this regard, the oocytes' maturity and their morphology can affect the efficacy of fertility preservation and ongoing fertilisation outcomes. The aim of this study was to evaluate the current options for female fertility preservation in emergency situations by cryopreservation of oocytes with different quality and maturity states.

\section{METHODS}

Literature searches were conducted in PubMed using the keywords "emergency fertility preservation", "fertility preservation for cancer patient", "oocyte vitrification", "oocyte in vitro maturation", and other words related to the current review. The studies were selected based on their relevance to the material presented in the review, the availability of the full text of the papers, as well as those that published the most recent data on the issue.

\section{Oocyte Cryopreservation}

In 2013, the American Society for Reproductive Medicine (ASRM) and the Society for Assisted Reproductive Technology (SART) published a joint document, 'Mature Oocyte Cryopreservation: A Guideline', which addressed advances in techniques to freeze human eggs that have resulted in significant recent improvements in pregnancy success. Based on the current state of evidence, modern procedures to cryopreserve oocytes should no longer be considered experimental. ${ }^{12}$ Since then, many more ART clinics around the world have actively started to offer oocyte cryopreservation as an effective method of preserving fertility. ${ }^{13-16}$

The fundamental science underpinning cryopreservation of female reproductive cells, including the basis for the two most frequently used techniques (controlled-rate slow-cooling or vitrification), is beyond the scope of this review and has been recently discussed. ${ }^{17,18}$ The first pregnancy was achieved following oocyte cryopreservation using the controlled-rate slow freezing method in 1986. ${ }^{19}$ After this, slow freezing has been considered the standard method for oocyte cryopreservation, and oocyte recovery of morphologically intact oocytes ranges from $50-60 \%$. The decrease in oocyte survival was associated with the formation of ice crystals during freezing and thawing. Currently, avoiding the formation of ice crystals around or inside oocytes has led to improved oocyte cryopreservation by 
the vitrification technique. The first pregnancy after oocyte vitrification was in 1999. ${ }^{20}$ However, there are still a large number of scientific studies comparing the effectiveness of the slow-cooling method and vitrification. ${ }^{21-23}$ Technological features of cryopreservation techniques do not determine the optimal method, since each has advantages and disadvantages (Box 1). However, different research findings and meta-analyses of oocyte survival rates, fertilisation outcomes, embryo development, and pregnancy rates have indicated that vitrification is a preferable method to slow freezing for mature oocytes (Table 1). These vitrification advantages can probably be explained by the enhanced preservation of the ultrastructural characteristics of oocytes. The data from transmission electron microscopy studies suggest that recovery of perivitelline spaces and mitochondria was more complete after vitrification than after slow freezing. ${ }^{27}$ The presence of normal oocyte meiotic spindle was greater after vitrification than after slow freezing (93.5\% versus $72.0 \% ; p=0.0128){ }^{28}$ Moreover, the gene expression profile of metaphase II (MII) oocytes is differentially affected by slow freezing and vitrification compared with noncryopreserved MII oocytes. ${ }^{29}$ Slow freezing was associated with downregulation of genes involved in chromosomal structure maintenance (KIF2C and KIF3A) and cell cycle regulation (CHEK2 and CDKN1B) that may lead to a reduction in the oocyte developmental competence after thawing compared with the vitrification procedure. This is confirmed by other data claiming that oocytes' potential to be fertilised and to develop into high-quality blastocysts was similar to embryos from fresh oocytes in oocyte donation programmes. ${ }^{30}$

Another challenge in mature oocyte cryopreservation is the presence of the meiotic spindle which consists of tubulin polymers sensitive to temperature fluctuation, along which the chromosomes are arranged ready for fertilisation. During cryopreservation, these microtubules can be depolymerised and cause impairment of proper chromosome segregation after thawing and fertilisation, such as nondisjunction, premature separation of sister chromatids, or the recently discussed reverse segregation which increases incidence of aneuploidies and possibly decreases mitochondrial activity. ${ }^{31}$ After thawing of vitrified oocytes, the meiotic spindle microtubules are able to repolymerise. ${ }^{32}$ The optimal time for this recovery depends on the cryopreservation method, the initial morphological and functional state of the oocytes, and the patient's age. ${ }^{33}$ It was shown that the meiotic spindle was visualised in only $35.7 \%$ of oocytes immediately after thawing. However, after 3 hours of incubation at $37{ }^{\circ} \mathrm{C}$ in a culture medium, the meiotic spindle was visualised in $57.4 \%$ of female gametes. ${ }^{34}$ It has been shown that meiotic spindle morphology and embryo ploidy are normally retained after oocyte vitrification and do not suffer negative impacts from the cryogenic cooling. ${ }^{35,36}$

Box 1: A comparison of oocyte cryopreservation methods.

\begin{tabular}{|l|l|l|}
\hline \multirow{2}{*}{} & \multicolumn{1}{|c|}{ Clow-cooling } & \multicolumn{1}{c|}{ Vitrification } \\
\cline { 2 - 4 } & \multicolumn{1}{|c|}{ Cryopreservation method } \\
\hline Advantages & $\begin{array}{l}\text { - Low cryoprotectant } \\
\text { concentration } \\
\text { - Controlled temperature steps }\end{array}$ & $\begin{array}{l}\text { - Not a time-consuming method; } \\
\text { - Cost-effective }\end{array}$ \\
\hline Disadvantages & $\begin{array}{l}\text { - Expensive specialised } \\
\text { equipment } \\
\text { - Time-consuming method }\end{array}$ & $\begin{array}{l}\text { - Cytotoxicity due to the high cryoprotectant } \\
\text { - Risk of cross-contamination between the samples } \\
\text { in liquid nitrogen in an open system } \\
\text { - Requires highly qualified specialists }\end{array}$ \\
\hline
\end{tabular}


Table 1: A comparison of study outcomes for oocyte cryopreservation methods.

\begin{tabular}{|c|c|c|}
\hline & \multicolumn{2}{|c|}{ Cryopreservation method } \\
\hline & Slow-cooling & Vitrification \\
\hline Survival rate & $\begin{array}{l}66.1 \%(298 / 451)(R R=1.23 ; 95 \% \mathrm{Cl}: 1.02-1.49 ; \\
\mathrm{p}<0.05) ; \\
\text { and three cohort studies (RR = 1.23; 95\% Cl: } \\
\text { 1.11-1.36; } \mathrm{p}<0.0001 \text { ) } \\
\text { (Rienzi L et al.. }{ }^{24} \text { 2017) }\end{array}$ & $\begin{array}{l}\text { 82.3\% (602/731) (RR: 1.23; 95\% Cl: 1.02-1.49; } \\
\text { p<0.05); } \\
\text { and three cohort studies (RR: 1.23; 95\% Cl: } \\
\text { 1.11-1.36; p<0.0001) } \\
\text { (Rienzi L et al.. }{ }^{24} 2017 \text { ) }\end{array}$ \\
\hline Fertilisation rate & $64.6 \%$ (Fadini R et al., ${ }^{25}$ 2009) & $72.8 \%$ (Fadini R et al., ${ }^{25}$ 2009) \\
\hline $\begin{array}{l}\text { Blastocyst- } \\
\text { formation rate }\end{array}$ & $12.0 \%$ (Cao YX et al., ${ }^{26}$ 2009) & $33.1 \%$ (Cao YX et al., ${ }^{26}$ 2009) \\
\hline $\begin{array}{l}\text { Clinical pregnancy } \\
\text { rate }\end{array}$ & 7.6\% (Fadini $\mathrm{R}$ et al., ${ }^{25}$ 2009) & 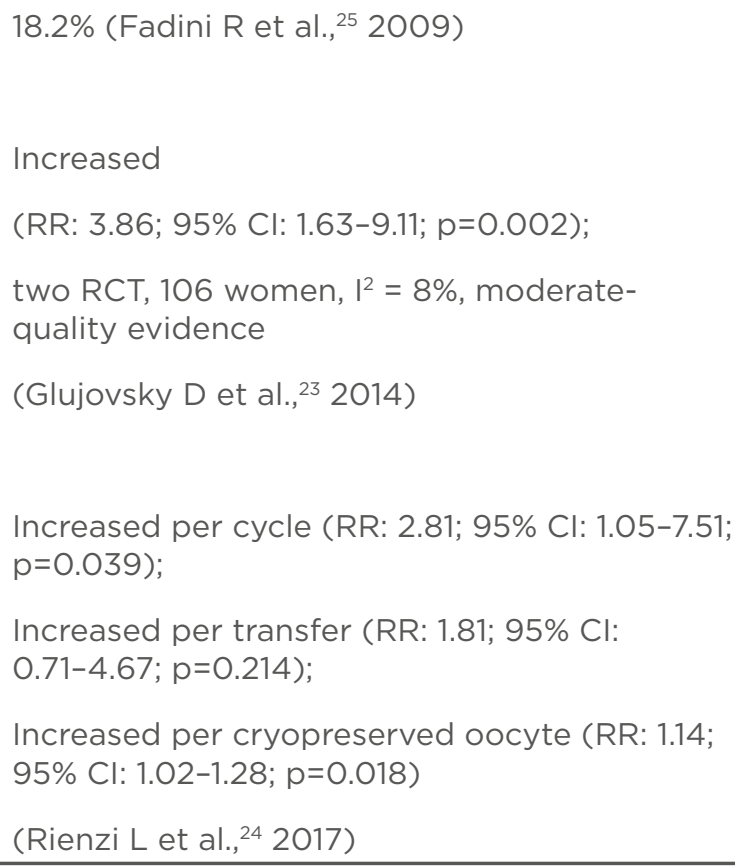 \\
\hline Implantation rate & $4.3 \%$ (Fadini $R$ et al., ${ }^{25} 2009$ ) & 9.3\% (Fadini $R$ et al.,25 2009) \\
\hline
\end{tabular}

$\mathrm{Cl}$ : confidence interval; RCT: randomised, controlled trials; RR: relative risk.

Another point to consider is oocyte morphological deviations, which exist among oocytes and may result from intrinsic factors such as maternal age and genetic defects or extrinsic factors such as stimulation protocols, culture conditions, and nutrition. These oocyte morphological features may affect the developmental competence and implantation potential of the derived embryo." They can also impact oocyte cryopreservation outcomes for individual cancer patients who wish to preserve their fertility. As with infertility services in general, counselling services for cancer patients who wish to engage with oocyte cryopreservation must be robust and informative, especially because decision making may need to progress quickly.

\section{Should All Aspirated Mature Oocytes Be Cryopreserved?}

According to the Istanbul consensus on the quality assessment of gametes and embryos, there are optimal morphological characteristics of oocytes: spherical structure; homogeneous zona pellucida (ZP); translucent, inclusions-free cytoplasm; and an adequate first polar body (PB) with no fragmentation sign. ${ }^{37}$ 
Oocyte structural abnormalities are divided into intra and extracytoplasmic. The first category includes different types and degrees of cytoplasmic granulations, variations in colour (dark-coloured cytoplasm, slightly diffused or excessive whole/centrally located granulation, lipofuscin bodies), the appearance of refractile bodies and smooth endoplasmic reticulum clusters, vacuolisation in the body of the oocyte, the maturity of the nucleus, and the presence of a normal meiotic spindle. The second category includes the presentation of the first PB (size, fragmentation) shape abnormalities (irregular shape of the MII oocyte), ZP abnormalities (dark or thick ZP), and perivitelline space abnormalities (large size, granularity). These morphological characteristics can be used as criteria for determining the quality of oocytes, and some may be predictors of their successful recovery after cryopreservation. ${ }^{38}$ ZP anomalies can arise from impaired secretion and glycoprotein matrix formation. ${ }^{39}$ Oocyte survival after cryopreservation depends on the morphological characteristics of ZP. The thickness can influence the cryoprotectant penetration; before cryopreservation, it is necessary to evaluate the morphological structure of the ZP and individually select the suitable time for cryoprotectant exposure, depending on the ZP thickness and oocyte osmotic reaction. ${ }^{40}$

The morphological structure of the first $\mathrm{PB}$ can be regarded as a reflection of the oocyte's post-vascular age. Oocytes with an oval PB and a smooth surface and without fragmentation develop into morphologically normal embryos with high implantation potential. The first-PB morphological abnormalities were associated with a decreased fertilisation rate, but did not show any correlation with embryo quality. ${ }^{41,42}$ The presence of an enlarged first PB is related to worse rates of fertilisation, cleavage, and highquality embryos. However, the identification of the first-PB fragmentation does not seem to interfere with the results of intracytoplasmic sperm injection, suggesting that oocyte selection based on PB fragmentation may not contribute to the identification of embryos with high developmental ability. It has been shown that not only the morphological characteristics of the first $\mathrm{PB}$, but also the angle of its location with respect to the pronuclei that appear after fertilisation, are important in predicting the quality of embryos. ${ }^{43}$
Oocyte first-PB morphology observed prior to vitrification can predict post-warming survival, and developing non-invasive identification of predictive markers for oocyte survival potential remains relevant.

Vacuoles are cytoplasmic inclusions filled with a fluid identical to the fluid of the perivitelline space. They are considered a morphological feature of the degenerative process in the oocyte because they can interfere with further fragmentation or division of the embryo in vitro, which in turn inhibits the rate of successful blastulation. $^{44}$ The presence of endoplasmic reticulum aggregation is associated with a lower chance of successful pregnancy and, even if normal oocytes are transferred from one pool, the pregnancy rate decreases. ${ }^{45}$ Despite the normal pregnancy rates obtained with oocytes with granular cytoplasm, $>50 \%$ of pregnancies resulted in miscarriages and the implantation rate was only $5 \% .{ }^{46}$ Variation in clustering and distribution of mitochondria can reflect developmental competence, and mitochondrial assessment can be used for analysis in vitrified or warmed oocytes. ${ }^{47}$

In addition to morphological features, oocytes can differ in their physical and chemical properties which can be observed when the suspending-medium osmolarity changes during cryopreservation processes. An individual osmotic response of oocytes may be considered flexible within the established range during the oocytes' exposure to low concentrations of cryoprotectants and to vitrification solutions. This approach does not contradict the recommendations of other investigations. The 'weaker' oocytes require a shorter equilibration time in cryoprotectant solution, whereas 'stronger' oocytes require prolonged exposure to the same solution for effective loading. ${ }^{48}$ Application of a flexible strategy helps to eliminate the risk of detrimental expansion or prolonged dehydration of cells, and the toxicological effects of cryopreservation media. This osmotic response during the equilibration procedure can be taken as an indicator of the oocyte's viability and may improve prediction of the cryopreservation outcomes; using a flexible time of exposure to cryoprotectant could increase the oocytes' survival. 
Development of criteria for assessment of mature oocytes is very important for predicting the outcomes of cryopreservation and possible individualisation of cryopreservation protocols, which are important when performing oocyte cryopreservation in emergency situations.

\section{'State of the Art' for Immature Oocyte Cryopreservation}

Obtaining immature oocytes in emergencies can be possible, even in situations where it may be necessary to eliminate costly drugs and frequent monitoring as used in routine ovarian stimulation; to complete treatment within 2-10 days, while avoiding the use of hormones in patients with hormone-sensitive tumours; and to retrieve oocytes at any point in the menstrual cycle, even in the luteal phase. In addition, immature oocytes can also be collected from extracorporeal ovarian biopsy specimens or ovaries during caesarean section. ${ }^{49}$ Emergency fertility preservation increases the number of cases of immature oocyte collection needing IVM. IVM programmes are already offered to poor responders or patients with polycystic ovary syndrome to avoid the risk of developing ovarian hyperstimulation syndrome caused by exogenous gonadotrophin stimulation. ${ }^{50}$ IVM protocols involve the addition of specific molecules to the in vitro oocyte culture media which have been implicated in reversing this meiosis-arresting action including cyclic adenosine monophosphate and purines. ${ }^{51,52}$ More than 5,000 babies have been born using IVM worldwide. ${ }^{53}$ The first baby from a vitrified IVM oocyte was reported in 2009.54 It is important not only that nuclear maturation progresses normally (reaching Stage MII), but that cytoplasmic (metabolic and structural changes in ooplasm) maturation also proceeds in step during IVM. Mammalian germinal vesicle (GV) stage immature oocytes can be divided into several types: nonsurrounded nucleolus (NSN); surrounded nucleolus (SN); partly NSN (pNSN) and SN (pSN); prematurely condensed NSN, pNSN, and pSN; and early diakinesis patterns. Maturation and embryo culture suggest that SN and pSN oocytes were more competent after fertilisation than NSN and pNSN oocytes; prematurely condensed pSN oocytes were more competent than prematurely condensed NSN/pNSN oocytes. ${ }^{55}$

One of the problems for the cryopreservation of immature oocytes is that oocytes are surrounded by a layer of cumulus cells which are essential participants in providing oocyte maturation and are sensitive to cryopreservation. ${ }^{56}$ Currently, the cryopreservation protocols for the oocyte-crowncumulus complex have not been successfully developed, meaning that oocytes must be released from these surrounding cells before cryopreservation. In this case, the connection between the oocyte and granulosa cells is disrupted which makes subsequent IVM more difficult. The outcomes of oocyte maturation and their subsequent fertilisation are dependent on the method of maturation. It was shown that supplementing growth hormone to the GV oocyte can lead, during in vitro culture, to an approximate $70.0 \%$ maturation rate. The fertilisation rate and rate of development to blastocyst were $73.1 \%$ and $25.0 \%$, respectively. ${ }^{57}$ These data confirm the promising use of GV oocytes for patient fertility preservation, which will be helpful in any requirement for emergency fertility preservation.

The problem of cryopreservation of immature oocytes raises the reasonable question of the best time to carry out cryopreservation: prior to or after in vitro maturation. However, several studies argue that the maturation rate was higher after vitrification at the GV stage. ${ }^{58}$ In contrast, other studies have shown that the maturation and fertilisation rates are significantly higher in the group of oocytes that were matured before cryopreservation. ${ }^{59-62}$ These results may be seen as paradoxical because mature oocytes at the MII stage have a meiotic spindle, which is extremely sensitive to temperature fluctuations and even insignificant fluctuations may cause depolymerisation of spindle microtubules, leading to chromosome segregation impairment, embryo aneuploidy, and developmental abnormalities. Moreover, vitrification can affect the GV oocyte ultrastructure; it was noted that there were significant changes in the structure of meiotic spindle of oocytes matured in vitro after vitrification. ${ }^{62,63}$ The current leading strategy for fertility preservation using the cryopreservation of immature oocytes should be development of gamete-maturation conditions that first allow for the obtainment of good-quality MII oocytes, followed by subsequent cryopreservation, with vitrification currently seen to be the most promising protocol. 


\section{FUTURE RESEARCH}

Experience in the cryopreservation of mature oocytes has been gained in elective practices Large, randomised, controlled trials are required to compare the various approaches for female fertility preservation to ensure the selection of an effective protocol. Currently, clinical trials databases have registered and tested trials comparing types of vitrification systems (open and closed) for oocytes and therapeutic intervention outcomes (primed either with recombinant human chorionic gonadotropin or a gonadotropin-releasing hormone agonist) prior to oocyte retrieval for IVM in patients with breast cancer. There have also been studies of outcomes for ovarian tissue cryopreservation following IVM or autologous transplantation of a retrieved oocyte. Further studies for IVM administration and the development of effective methods for ovarian tissue cryopreservation are still required.

\section{CONCLUSIONS}

Although oocyte cryopreservation provides many benefits for fertility preservation in general, its importance for emergency fertility preservation has not been widely discussed. such as IVF; however, it is necessary to develop evaluation criteria to predict the effectiveness of oocyte cryopreservation and application of individualised oocyte cryopreservation protocols. Using immature oocytes for in vitro maturation and cryopreservation is promising for patients requiring emergency fertility preservation; however, further studies of this process, and development of enhanced maturation and vitrification techniques, are necessary. In addition, the results at one clinic of the $15^{\text {th }}$ year cryopreservation of oocytes for patients with cancer showed that only $4.5 \%$ patients returned to use their gametes. ${ }^{64}$ In the future, cryopreservation of ovarian tissues or isolated ovarian follicles may also contribute significantly to fertility preservation in emergency situations. Nevertheless, offering fertility preservation is no longer considered optional and must be included in every therapeutic programme for women who receive an oncological diagnosis in their reproductive age. This in turn will require development of close collaborations between cancer and fertility centres to provide a holistic, patient-centered fertility preservation strategy for female oncological patients.

\section{References}

1. Pampanini $\vee$ et al. Impact of firstline cancer treatment on the follicle quality in cryopreserved ovarian samples from girls and young women. Hum Reprod. 2019;34(9):1674-85.

2. Tesarik J. After corona: there is life after the pandemic. Reprod Biomed Online. 2020;40(6):760-2.

3. Ferlay $\mathrm{J}$ et al. Cancer incidence and mortality patterns in Europe: estimates for 40 countries and 25 major cancers in 2018. Eur J Cancer 2018;103:356-87.

4. Salama M, Mallmann P. Emergency fertility preservation for female patients with cancer: clinical perspectives. Anticancer Res. 2015:35(6):3117-27.

5. Fasano $\mathrm{G}$ et al. Outcomes of immature oocytes collected from ovarian tissue for cryopreservation in adult and prepubertal patients. Reprod Biomed Online. 2017;34(6):575-82

6. Muñoz E et al. Ovarian stimulation in patients with breast cancer. Ecancermedicalscience. 2015;9:504.
7. Moravek MB et al. Long-term outcomes in cancer patients who did or did not pursue fertility preservation. Fertil Steril. 2018;109(2):349-55.

8. Leonel ECR et al. Cryopreservation of human ovarian tissue: a review. Transfus Med Hemother. 2019;46(3):173-81.

9. McLaughlin M et al. Metaphase II oocytes from human unilaminar follicles grown in a multi-step culture system. Mol Hum Reprod. 2018;24(3):135-42

10. Grynberg $M$ et al. Similar in vitro maturation rates of oocytes retrieved during the follicular or luteal phase offer flexible options for urgent fertility preservation in breast cancer patients. Hum Reprod. 2016;31(3):623-9.

11. Balaban B, Urman B. Effect of oocyte morphology on embryo development and implantation. Reprod Biomed Online. 2006;12(5):608-15.

12. Noyes $\mathrm{N}$ et al. Oocyte cryopreservation: is it time to remove its experimental label? J Assist Reprod Genet. 2010;27(2-3):69-74

13. Cobo A et al. Comparison of concomitant outcome achieved with fresh and cryopreserved donor oocytes vitrified by the Cryotop method. Fertil Steril. 2008;89:165764.

14. Cobo A et al. Use of cryo-banked oocytes in an ovum donation programme: a prospective, randomized, controlled, clinical trial. Hum Reprod. 2010;25(9):2239-46.

15. Rienzi L et al. Embryo development of fresh 'versus' vitrified metaphase II oocytes after ICSI: a prospective randomized sibling-oocyte study. Hum Reprod. 2010;25(1):66-73.

16. Parmegiani $L$ et al. Efficiency of aseptic open vitrification and hermetical cryostorage of human oocytes. Reprod Biomed Online. 2011;23(4):505-12.

17. Yurchuk $T$ et al. Science of cryopreservation in reproductive medicine - embryos and oocytes as exemplars. Early Hum Dev. 
2018;126:6-9.

18. Rienzi LF et al. Perspectives in gamete and embryo cryopreservation. Semin Reprod Med. 2018;36(5):253-64.

19. Chen C. Pregnancy after human oocyte cryopreservation. Lancet. 1986;1(8486):884--6.

20. Kuleshova $L$ et al. Birth following vitrification of a small number of human oocytes: case report. Hum Reprod. 1999;14(12):3077-9.

21. Levi-Setti PE et al. Evolution of human oocyte cryopreservation: slow freezing versus vitrification. Curr Opin Endocrinol Diabetes Obes. 2016;23(6) 445-50.

22. Youm HS et al. Vitrfication and slow freezing for cryopreservation of germinal vesicle-stage human oocytes: a Bayesian meta-analysis. Cryo Letters. 2017;38(6):455-62.

23. Glujovsky D et al. Vitrification versus slow freezing for women undergoing oocyte cryopreservation. Cochrane Database Syst Rev. 2014;(9):CD010047.

24. Rienzi L et al. Oocyte, embryo and blastocyst cryopreservation in ART: systematic review and meta-analysis comparing slow-freezing versus vitrification to produce evidence for the development of global guidance. Hum Reprod Update. 2017;23(2):13955.

25. Fadini R et al. Human oocyte cryopreservation: comparison between slow and ultrarapid methods. Reprod Biomed Online. 2009;19(2):171-80. Erratum in: Reprod Biomed Online. 2009;19(5):757.

26. Cao YX et al. Comparison of survival and embryonic development in human oocytes cryopreserved by slow-freezing and vitrification. Fertil Steril. 2009;92(4):1306-11.

27. Li YZ et al. Vitrification versus slow freezing of human oocytes: effects on ultrastructure and developmental potential. Reprod Dev Med. 2018;2(3);129-36.

28. Martínez-Burgos $M$ et al. Vitrification versus slow freezing of oocytes: effects on morphologic appearance, meiotic spindle configuration, and DNA damage. Fertil Steril. 2011;95(1):374-7.

29. Monzo $C$ et al. Slow freezing and vitrification differentially modify the gene expression profile of human metaphase II oocytes. Hum Reprod. 2012;27(7):2160-8.

30. García Jl et al. Efficacy of oocyte vitrification combined with blastocyst stage transfer in an egg donation program. Hum Reprod. 2011;26(4):782-90.

31. Cimadomo D et al. Impact of maternal age on oocyte and embryo competence. Front Endocrinol
(Lausanne). 2018;9:327.

32. Tamura AN et al. Impact of vitrification on the meiotic spindle and components of the microtubuleorganizing center in mouse mature oocytes. Biol Reprod. 2013;89(5):112.

33. Asgari $\vee$ et al. Time dependent effect of post warming interval on microtubule organization, meiotic status, and parthenogenetic activation of vitrified in vitro matured sheep oocytes. Theriogenology. 2011;75(5):904-10.

34. Rienzi $L$ et al. Polscope analysis of meiotic spindle changes in living metaphase II human oocytes during the freezing and thawing procedures. Hum Reprod. 2004;19(3):655-9.

35. Buderatska $\mathrm{N}$ et al. Does human oocyte cryopreservation effect equally on embryo chromosome aneuploidy? Cryobiology. 2020;93:336

36. De Munck $\mathrm{N}$ et al. Chromosomal meiotic segregation, embryonic developmental kinetics and DNA (hydroxy)methylation analysis consolidate the safety of human oocyte vitrification. Mol Hum Reprod. 2015;21(6):535-44.

37. Alpha scientists in reproductive medicine and ESHRE special interest group of embryology. The Istanbul consensus workshop on embryo assessment: proceedings of an expert meeting. Hum Reprod. 2011;26(6):1270-83.

38. Rienzi $L$ et al. Predictive value of oocyte morphology in human IVF: a systematic review of the literature. Hum Reprod Update. 2010;17(1):3445.

39. Choi JK et al. The crucial role of zona pellucida in cryopreservation of oocytes by vitrification. Cryobiology. 2015; 71(2):350-5.

40. Petrushko MP et al. Individualization of the cryopreservation procedure of biopsied embryos allows to increase their survival. Cryobiology. 2018;85:181-82

41. Rienzi $L$ et al. Significance of metaphase II human oocyte morphology on ICSI outcome. Fertil Steril. 2008;90(5):1692-700.

42. Navarro PA et al. Relationship between first polar body morphology before intracytoplasmic sperm injection and fertilization rate, cleavage rate, and embryo quality. Int J Gynaecol Obstet. 2009;104(3):226-

43. Setti AS et al. Oocyte morphology does not affect post-warming survival rate in an egg-cryobanking donation program. J Assist Reprod Genet. 2011:28(12):1177-81.

44. Petrushko $M$ et al. Cryopreservation of incomplete compacted morulae and preliminary biopsy of excluded fragments. Zygote. 2019;27(6):386-91.
45. Otsuki J et al. The relationship between pregnancy outcome and smooth endoplasmic reticulum clusters in MII human oocytes. Hum Reprod. 2004;19(7):1591-7.

46. Kahraman S et al. Relationship between granular cytoplasm of oocytes and pregnancy outcome following intracytoplasmic sperm injection. Hum Reprod. 2000;15(11):2390-3.

47. Nohales-Córcoles $M$ et al. Impact of vitrification on the mitochondrial activity and redox homeostasis of human oocyte. Hum Reprod. 2016;31(8):1850-8.

48. Son WY et al. Immature oocyte for fertility preservation. Front Endocrinol (Lausanne). 2019;10:464

49. Cha KY et al. Obstetric outcome of patients with polycystic ovary syndrome treated by in vitro maturation and in vitro fertilizationembryo transfer. Fertil Steril. 2005;83(5):1461-5.

50. Eppig JJ et al. Chemical signals that regulate mammalian oocyte maturation. Biol Reprod 1984;30(1):1-

51. Downs SM et al. Hypoxanthine is the principal inhibitor of murine oocyte maturation in a low molecular weight fraction of porcine follicular fluid. Proc Natl Acad Sci U S A. 1985;82(2):454-8.

52. Sauerbrun-Cutler MT et al. In vitro maturation and its role in clinical assisted reproductive technology. Obstet Gynecol Surv. 2015;70(1):4557.

53. Chian RC et al. Live birth after vitrification of in vitro matured human oocytes. Fertil Steril. 2009;91(2):3726.

54. Pan $L Z$ et al. A new classification of the germinal vesicle chromatin configurations in pig oocytes. Biol Reprod. 2018;99(6):1149-58.

55. Li $Y$ et al. Growth hormone promotes in vitro maturation of human oocytes. Front Endocrinol (Lausanne). 2019;10:485.

56. Ruppert-Lingham CJ et al. Membrane integrity and development of immature murine cumulus-oocyte complexes following slow cooling to -60 degrees $\mathrm{C}$ : the effect of immediate rewarming, plunging into LN2 and two-controlled-rate-stage cooling. Cryobiology. 2006;52(2):21927.

57. Molina I et al. Osmotic-shock produced by vitrification solutions improves immature human oocytes in vitro maturation. Reprod Biol Endocrinol. 2016;14(1):27.

58. Fasano $\mathrm{G}$ et al. In-vitro maturation of human oocytes: before or after vitrification? J Assist Reprod Genet. 2012;29(6):507-12. 
59. Lee JA et al. In-vitro maturation of germinal vesicle and metaphase I eggs prior to cryopreservation optimizes reproductive potential in patients undergoing fertility preservation. Curr Opin Obstet Gynecol. 2014;26(3):168-73.

60. Lee JA et al. Optimizing human oocyte cryopreservation for fertility preservation patients: should we mature then freeze or freeze then mature? Fertil Steril. 2013;99(5):135662.

61. Cao $\mathrm{Y}$ et al. Cryopreservation of immature and in-vitro matured human oocytes by vitrification. Reprod Biomed Online. 2009;19(3):369-73.

62. Liu $\mathrm{MH}$ et al. Ultrastructural changes and methylation of human oocytes vitrified at the germinal vesicle stage and matured in vitro after thawing. Gynecol Obstet Invest.
2017:82(3):252-61.

63. Shahedi A et al. The effect of vitrification on ultrastructure of human in vitro matured germinal vesicle oocytes. Eur J Obstet Gynecol Reprod Biol. 2013;167(1):69-75.

64. Specchia $\mathrm{C}$ et al. Oocyte cryopreservation in oncological patients: eighteen years experience of a tertiary care referral center. Front Endocrinol (Lausanne). 2019;10:600. 